\title{
An Automated Negotiation Model for M-commerce Using Mobile Agents
}

\author{
Fernando Menezes Matos and Edmundo R.M. Madeira \\ IC - Institute of Computing \\ UNICAMP - University of Campinas \\ 13083-970 Campinas, SP, Brazil \\ \{fernando.matos, edmundo\}@ic.unicamp.br
}

\begin{abstract}
This work proposes an automated negotiation model between two participants, for m-commerce, using mobile agents and considering the mobile device personalization through the use of profiles in the negotiation.
\end{abstract}

Keywords: M-commerce, Mobile Agents, Automated Negotiation

\section{Introduction}

Due to the growing number of cellular phones and PDAs, more and more people desire to perform activities using them. One of these activities is mobile commerce (m-commerce), where a user can buy a product or service no matter where he is.

However, to do this it is necessary to develop techniques and communication mechanisms for mobile commerce, which satisfy the requirements and the limitations of the mobile infrastructure, like the reduced screen size of the cellular phones and the low bandwidth of the wireless networks [1.

Another issue to take into account in mobile commerce is the fact that mobile devices are personal. Due to this, it is interesting the development and use of a user profile. If the transactions could be performed using this profile, they will become more similar to what is done in the real life, guaranteeing a great purchase personalization.

This paper proposes an automated negotiation model for mobile commerce using mobile agents based on a preexisting negotiation model for electronic commerce [2]. The extensions presented here, take into account the low processing power of the portable devices, the small screen size of these devices, the low bandwidth of the wireless networks and mainly the user profile.

\section{The Negotiation Model for Mobile Commerce}

\subsection{Model}

The model works as follows. There is an application in the mobile device, called BuyApplication, used by the client to make purchases. Each time a mobile network user wishes to make a purchase, BuyApplication connects to another application called CallApplication, which is hosted in a server in the fixed network, 
and sends information about the product which the user wants, like its features, the desired price and the maximum price to offer. After that, CallApplication creates an original buy agent for each incoming request. Fig. 11a) shows the creation of $n$ original buy agents due to $n$ buy requests. Each original buy agent creates a certain number of buy agent copies to negotiate with the sell agents. Fig. 1(b) illustrates that the original buy agent A1 created two buy agent copies that negotiate with two sell agents, related to an incoming request. The proportion between sell agents and buy agents is one to one

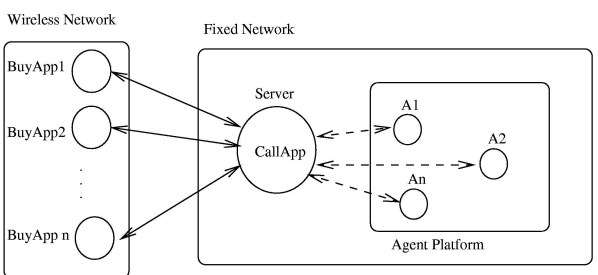

(a)

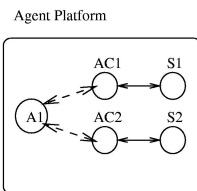

(b)

Fig. 1. Communication in the Proposed Model.

After the buy agents have been created, they move to the host of the selected sellers to negotiate with them. Once there, they start to negotiate until they reach an agreement, or one party rejects the other's offer. At the end of the negotiation, each buy agent sends its results to the original buy agent to present to the user.

\subsection{Profiles}

One of the inherent features of mobile devices is their customization. Hence, the creation of a user profile for mobile commerce is important. In this work, we have created two types of profiles. The first one is related to the user and contains the following items:

Number of Negotiations: this item defines the maximum number of buy agent copies created by the original buy agent to negotiate;

Number of Results: this item defines the maximum number of responses that will return to the user;

Automatic Purchase: this item defines if the original buy agent must perform the purchase after all results have returned, without previous consultation to the user.

The other profile type is the purchase profile and contains the following items:

Fast Negotiation: if this item is enabled, the original buy agent performs the purchase of the first incoming result below the desired price plus a percentage (variation), without informing the user about the negotiation results, and it informs all other buy agents to stop the negotiation; 
Fidelity: this item defines if the user wants to keep fidelity to a seller. The original buy agent performs the purchase of the first incoming result if its price is lower than the desired price plus a percentage and if it is a result from that specific seller;

Connected: this item defines if the user wishes to keep connected to the server during the negotiation. In this case, the original buy agent informs the user about the results. Otherwise, information of any pending negotiation is recorded in the fixed network, and made available when the user reconnects. Then, the user can establish connection with the original buy agent responsible for his pending negotiation;

Variation: this item defines a maximum percentage over the desired price to offer in negotiations performed involving the items Fast Negotiation and Fidelity of the purchase profile.

\subsection{Phases}

The proposed model for mobile commerce is divided in 7 phases.

Connection Phase: when the user wants to start a new negotiation, he sends a message to the fixed network, requesting a new negotiation process. This message contains the desired product price and its features. When the request arrives, the application in the fixed network creates an original buy agent responsible for performing the negotiation.

Call Phase: in this phase a multicast message is sent by the original buy agent to the sell agents, in order to verify which of them have the desired product.

Selection Phase: in this phase, the original buy agent selects which sell agents it will negotiate with, amongst the sell agents which responded affirmatively in the prior phase.

Negotiation Phase: in this phase, a copy of the original buy agent is sent for each host of the sell agents selected in the previous phase. After that, the price negotiation is performed. This is done using proposals and counterproposals between the agents. The agreement between the agents occurs when the price offered by the sell agent is lower than or equal to the price proposed by the buy agent. An agreement finishes the negotiation. Another way to terminate the negotiation is when the buy agent should propose a price higher than the maximum the client would pay.

After an agreement is reached, the buy agent requests a discount to the sell agent upon the agreed price, through the Discount message. The sell agent may grant the discount or not, depending if it is using any fidelity service and if the buyer is its client or not.

Analysis Phase: this is the phase where decisions are made taking into account almost every information contained in the user and purchase profiles. The possibilities are as follows.

Fast Negotiation: The original buy agent doesn't wait for the return of all responses. When the first result from a buy agent, in which the obtained price is lower than the desired price plus a percentage (defined in the user profile by the variation item) returns, the original buy agent performs 
the purchase and informs all other buy agents to stop the negotiation. This kind of negotiation is extremely useful in mobile commerce since mobile devices have little autonomy due to its dependency on batteries;

Fidelity: if this item is enabled, when the first result in which the obtained price is lower than the desired price plus a percentage (defined in the user profile by the variation item) returns and the result came from a seller the user wishes to keep fidelity to, the original buy agent performs the purchase;

Automatic Purchase: if this option is enabled, the original buy agent waits the return of all responses and then it asks for the copy that got the lower price to perform the purchase. If it is not enabled, the original buy agent sorts the offers in increasing order of price and sends them to the application hosted in the mobile device to be presented to the user, considering the number of results defined by the user.

Presentation Phase: this phase starts when an original buy agent has all results and it has already taken the decisions about them, using the user and purchase profiles. If the Connected item is enabled, the agent sends the results to the user. Otherwise, it waits for the user to establish a connection and to request the results.

Conclusion Phase: in this phase the negotiation is concluded. Either the user chooses one of the presented proposals or is informed about the purchase that was performed.

\section{Conclusion}

This paper proposes an automated negotiation model, using mobile agents, for mobile commerce. The contributions are: a-) the definition of user and purchase profiles. That are used mainly in the Analysis phase to make decisions about the product purchase; b-) all the heavy computation is performed in the fixed network, by the CallApplication and the agent platform, and do not overload the mobile device. It also does not overload the communication channel, because it imposes a small data traffic between the mobile device and the fixed network; and c-) the user doesn't need to be connected during the negotiation, since he can request the results afterward.

\section{References}

1. Tsalgatidou, A., Veijalainen, J., Pitoura, E.: Challenges in Mobile Electronic Commerce. In: Proceedings of Iec 2000. Third Internacional Conference on Innovation through E-Commerce. Manchester England (2000) 169-174

2. Ueyama, J., Madeira, E.R.M.: An Automated Negotiation Model for Electronic Commerce. In: Proceedings of the Fifth IEEE Internationl Symposium on Autonomous Decentralized Systems - ISADS'01. Dallas USA (2001) 29-36 